\title{
Olfactory improvement in acromegaly after transnasal transsphenoidal surgery
}

\author{
Bertrand Actor, Med. Pract., ${ }^{1}$ Johannes Sarnthein, Ph.D., ${ }^{1}$ \\ Peter Prömmel, Med. Pract., ${ }^{1}$ David Holzmann, M.D., ${ }^{2}$ and René L. Bernays, M.D. ${ }^{1}$ \\ Departments of ${ }^{1}$ Neurosurgery and ${ }^{2}$ Otorhinolaryngology, University Hospital Zurich, Switzerland
}

\begin{abstract}
Object. The direct transnasal transsphenoidal approach to the sellar region has become a widely adopted surgical procedure among neurosurgeons and ear, nose, and throat specialists. Nasal complications and their incidence have been investigated, but a systematic testing of olfactory disturbance has not previously been performed. Considering that the sense of smell is deeply anchored and interwoven within the CNS, and that its impairment implies a considerable loss in quality of life, surgical practice should aim at its preservation.

Methods In this retrospective study, pre- and postoperative olfactory performance, nasal airway passage, septal perforation, and epistaxis were assessed in 96 patients who underwent direct transnasal transsphenoidal microsurgery at the authors' department between January 2007 and August 2009. Olfactory performance was assessed using the Sniffin' Sticks test and/or the Zürcher Geruchstest.

Results. After surgery, 47 (49\%) of 96 patients improved, 34 (35\%) of 96 deteriorated, and 15 (16\%) of 96 presented with unchanged olfactory performance. With respect to the underlying pathological entity, the authors noticed a remarkable difference between patients with acromegaly ( 23 cases) and all other patients ( 73 cases). Fifteen ( $65 \%$ ) of 23 patients with acromegaly improved (others $44 \%$ ), only $3(13 \%)$ of 23 deteriorated (others $42 \%)$, and $5(22 \%)$ of 23 remained unchanged (others $14 \%$ ) in their ability to distinguish odors. This illustrates a significant shift toward improved postoperative olfactory performance (cross-tabulation, Fisher exact test; $p=0.028$ ) in patients with acromegaly.

In nasal breathing, $77(80 \%)$ of 96 patients noticed no change, $11(12 \%)$ of 96 improved, and $8(8 \%)$ of 96 worsened postoperatively. Of the 11 patients with improved breathing, $6(55 \%)$ had acromegaly. Improved nasal airway patency was more frequent in patients with acromegaly (cross-tabulation, Fisher exact test; $\mathrm{p}=0.002$ ).

Conclusions. The data provide the first significant evidence for improvement in olfactory performance in patients with acromegaly after transsphenoidal surgery (TSS) of growth hormone-producing adenomas. Furthermore, postoperative olfactory disturbance in patients treated with transnasal TSS is more frequent than previously reported. Nevertheless, recurrent transnasal TSS can be performed successfully, even multiple times, and does not involve a higher risk of nasal complications. (DOI: 10.3171/2010.7.FOCUS10162)
\end{abstract}

\section{KEY WORDS $\bullet \quad$ nasal complication $\bullet \quad$ transnasal approach $\bullet$
transsphenoidal surgery $\bullet$ olfaction $\bullet \quad$ acromegaly $\bullet$ growth hormone}

$\mathrm{T}$ HE transsphenoidal approach to the sella turcica is the most popular technique for the resection of pituitary adenomas and other tumors of the sellar region. Throughout its implementation, various access routes have been developed, including the sublabial transseptal, transantral, transethmoidal, and especially with the improvement of endoscopic methods, the direct transnasal access has become a routine standard in pituitary surgery. Since the year 2000, the direct transnasal approach to the sphenoid sinus, as first described by Griffith and Veerapen ${ }^{5}$ in 1987, has been applied for TSS at our department. Nasal

Abbreviations used in this paper: $\mathrm{GH}=$ growth hormone; $\mathrm{TSS}=$ transsphenoidal surgery. complications caused by this approach have previously been described, but olfactory disturbance has never been investigated in a systematic fashion. The consideration that the sense of smell is deeply anchored and interwoven within the CNS, and that the coding of its function requires $1 \%$ of our genome, ${ }^{2}$ yields ample incentive for its preservation. These circumstances have motivated us to explore olfactory complications in this widely established surgical approach systematically, to allow more precise counseling of patients prior to their consent to an operation.

A further goal of the study was to test our hypothesis that olfactory performance might improve due to surgery, similar to improvement in median nerve neuropathy after treatment of acromegaly? 


\section{Methods}

\section{Patient Inclusion Criteria}

A retrospective study was performed in all patients who underwent transnasal TSS at our department between January 2007 and August 2009. A total of 145 operations, excluding revision operations, were performed in 141 patients by the senior author (R.L.B.). At our department, olfactory testing is performed as part of the clinical evaluation in patients who are scheduled to have TSS. All patients who received preoperative olfactory testing were included in the evaluation. These criteria led to a final group of 96 patients who answered questions concerning nasal obstruction, underwent examination of the nasal septum, and were retested in regard to olfactory function at follow-up evaluation.

\section{Preoperative Assessment}

All 96 patients underwent preoperative testing of olfactory performance in our department or at our local ear, nose, and throat department. Testing was done with the Sniffin' Sticks olfactory test (Burghart Medizintechnik) or Zürcher Geruchstest (www.novimed.ch). In olfactory testing, both sides were investigated simultaneously. Correct recognition of more than $85 \%$ of presented odors was defined as normal, between $85 \%$ and $50 \%$ as hyposmic, and below $50 \%$ as anosmic. The nasal septum was examined at the beginning of each operation by the senior author.

\section{Surgical Technique}

Microsurgery was performed in the PoleStar N20 apparatus $(0.15 \mathrm{~T}$, www.medtronic.com) by means of a direct transnasal transsphenoidal approach. Following orotracheal induction of anesthesia, the patient was placed in a supine position with the head reclined and fixed in the head holder. The nasal cavities were packed for 3-5 minutes with gauze soaked in a mixture of $30 \mathrm{ml} 2 \%$ tetracaine $\mathrm{HCl}$ and $2 \mathrm{mg}$ adrenaline. Nostrils and surrounding skin were then disinfected with iodine solution. After draping, a self-retaining nasal speculum (www.aesculap.com) was placed in the nasal cavity, typically the narrower one, and the anterior sphenoid wall was visualized with the aid of the operating microscope. The posterior part of the septal bone was then fractured toward the opposite side by leverage with the speculum, and the mucosa was incised in an inverted T shape with a disc knife. The first speculum was withdrawn, and a Buchfelder pituitary speculum (www. dewimed.de) was inserted and fixed in position. After that, the anterior sphenoid wall was removed with punches or a chisel to gain access to the sphenoid sinus. Finally, the sellar pathological entity was removed, without postoperative packing of the nasal cavities.

\section{Postoperative Treatment and Follow-Up}

Following the operation, a CT scan was obtained to rule out postoperative complications, and patients were observed daily for fluid and electrolyte balance, epistaxis, nasal dripping, and visual acuity. Acromegaly was considered cured if the GH serum level dropped below $1 \mu \mathrm{g} / \mathrm{L}$, as defined in the acromegaly treatment consensus workshop. ${ }^{1,10}$ Patients with epistaxis were clinically examined if the bleeding was immediately pronounced or if it did not subside within 1 to 2 days of surgery. Postoperative nasal crusting and/or clotting was gently resolved using an isotonic, sterile, saltwater nose spray (Triomer spray, Vifor AG) 3-4 times daily.

After discharge, patients typically received clinical follow-up and underwent MR imaging 3 months postoperatively, and thereafter in yearly intervals. At follow-up, the patients' subjective perception of postoperative nasal breathing ability was assessed in comparison with before surgery. Furthermore, nasal septum defects were evaluated using rhinoscopy, and olfactory performance was tested only with the Zürcher Geruchstest by the senior author.

\section{Statistical Analysis}

At each olfactory testing session, the number of correctly assigned odors was transformed into an olfactory performance rate, with a range of $0 \%-100 \%$. Statistical testing was performed using the PASW Statistics 18 program. For cross-tabulation, we used the Fisher exact test because of small cell counts. Because our data cannot be assumed to be normally distributed, we applied nonparametric testing: the Mann-Whitney test for comparisons between groups and the paired Wilcoxon signed-ranks test for before and after comparisons within groups. All tests were 2-sided, and the significance level was set at 0.05 .

\section{Results}

\section{Patient Demographic Data}

In the 32-month period, 96 patients who underwent 98 direct transnasal transsphenoidal operations were included in this study. The age range was $11.9-86.7$ years (mean 53.1 years). There were 62 male and 34 female patients. Two participants had repeated surgery within these 32 months: a 19-year-old woman with recurrence of a GH-producing macroadenoma, and a 50-year-old man with recurrent Rathke cyst. The cohort included 72 macroadenomas, 11 microadenomas, 9 Rathke cysts, and 1 each of meningioma, craniopharyngioma, arachnoid cyst, and oncocytoma. Among the adenomas were 41 hormone-inactive and 42 hormone-active tumors. Of the 96 patients, 23 (24\%) had acromegaly; 18 of them were considered cured according to the definition of the acromegaly consensus workshop, ${ }^{1,10}$ 4 had normal serum GH levels (but $>1 \mu \mathrm{g} / \mathrm{L}$ ), and 1 displayed an elevated level of $6.2 \mu \mathrm{g} / \mathrm{L}$ at follow-up. Overall there were 17 recurrent tumors (18\%), 16 of which had been previously treated transsphenoidally (Table 1) and 1 transcranially at another center. Follow-up duration ranged from 3 to 34 months (mean 10.3 months).

\section{Olfactory Performance}

Preoperatively, 39 (41\%) of 96 patients displayed normal smelling capability, 53 (55\%) had hyposmia, and 4 (4\%) were anosmic. Postoperatively, 60 (62\%) of 96 patients had normal olfactory performance, 30 (31\%) were hyposmic, and $6(6 \%)$ had anosmia. Comparing pre- and 


\section{TABLE 1: Overview of prior treatments in 17 patients with recurrent disease*}

\begin{tabular}{cll}
\hline $\begin{array}{c}\text { No. of } \\
\text { Cases }\end{array}$ & Type of Recurrent Disease & No. \& Type of Prior Treatments \\
\hline 7 & inactive macroadenoma & TSS (1) \\
3 & active macroadenoma & TSS (1) \\
1 & inactive macroadenoma & TSS (2), TCS (1), RT (1) \\
1 & inactive macroadenoma & TSS (5), RS (1) \\
1 & active macroadenoma & TSS (1), RS (1) \\
1 & inactive macroadenoma & TSS (3) \\
1 & Rathke cyst & TSS (2) \\
1 & Rathke cyst & TSS (1) \\
1 & inactive macroadenoma & TCS (1) \\
\hline
\end{tabular}

* Numbers in parentheses represent the number of treatments. Abbreviations: $\mathrm{RS}=$ radiosurgery (Gamma Knife, linear accelerator); RT = radiotherapy; TCS $=$ transcranial surgery.

postoperative performance of each patient, we found 15 (16\%) of 96 to have experienced no change in their smelling capability (unchanged group), 47 (49\%) were able to discriminate more odors (improved group), and 34 (35\%) had deteriorated in their ability to distinguish the tested substances (deteriorated group).

Comparing the groups with improved, deteriorated, and unchanged olfaction divided according to the underlying disease, we noticed a remarkable difference between the 23 patients with acromegaly and the 73 others. Among the patients with acromegaly, 15 (65\%) of 23 improved, 3 $(13 \%)$ deteriorated, and $5(22 \%)$ remained unchanged. The values differ remarkably from the patients without acromegaly, of whom 32 (44\%) of 73 improved, 31 (42\%) deteriorated, and 10 (14\%) remained unchanged. The num- bers are illustrated in Fig. 1. Based on these numbers, we constructed a table for the categories "olfaction improved/ unchanged/deteriorated" and "acromegalic/nonacromegalic," and found a significant difference for the effect of surgery between the 2 patient groups (Fisher exact test; $\mathrm{p}=$ 0.028 ). This difference between the groups was confirmed by a nonparametric comparison (Mann-Whitney test; $\mathrm{p}=$ 0.025 ). We then compared olfactory performance rates preand postoperatively within each group (paired Wilcoxon test). A significant improvement was only found in the acromegalic group $(\mathrm{p}=0.009)$, and not in the nonacromegalic group ( $\mathrm{p}=0.9)$. This is reflected in the median change of olfactory performance in the 2 groups: acromegalic ( $8 \%$, range from $-16 \%$ to $33 \%$ ), and nonacromegalic $(0 \%$, range from $-67 \%$ to $63 \%$ ). This reflects a change of noticeable magnitude pointing toward its clinical relevance.

\section{Nasal Airway Patency}

Unaffected, normal nasal respiration was reported in $77(80 \%)$ of 96 cases. Better nasal air passage was reported in $11(12 \%)$ of 96 cases, and $8(8 \%)$ of 96 patients expressed reduced patency after the operation. Two of these 8 experienced an overall reduced ability to breathe through their nose, whereas 3 each were able to state the side of dominant obstruction. In the 11 cases with better postoperative air passage, 6 patients $(55 \%)$ had GH-producing adenomas and 1 patient had a correction of a nasal septal deviation in combination with the TSS.

Surgery affected the airway patency of patients with and without acromegaly differently. Of the patients with acromegaly, 3 (13\%) of 23 deteriorated, $13(57 \%)$ remained unchanged, and 7 (30\%) improved. Of the nonacromegalic patients, $5(7 \%)$ of 73 deteriorated, $64(88 \%)$ remained unchanged, and $4(6 \%)$ improved. The difference between patient groups was significant (Fisher exact test; $\mathrm{p}=0.002$ ).

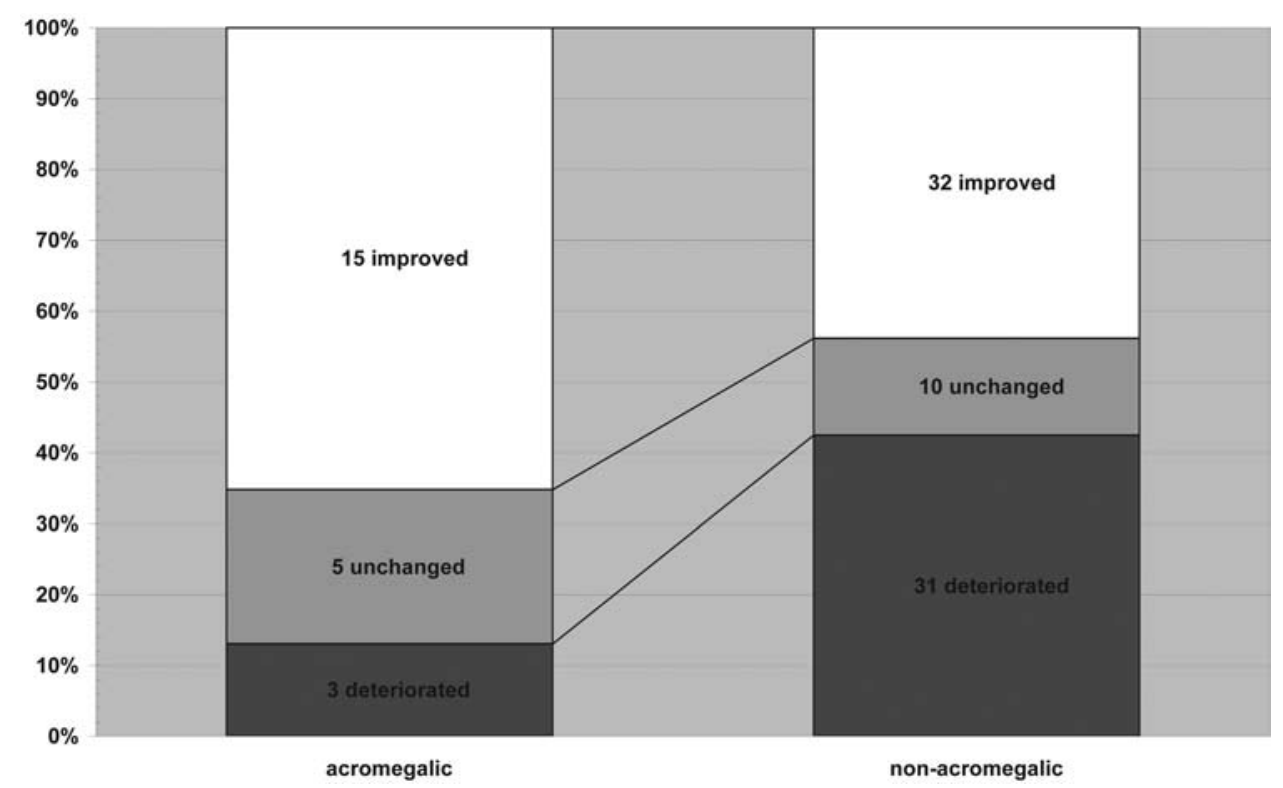

FIG. 1. Bar graph displaying olfactory performance after surgery in patients with compared with those without acromegaly. The percentages of patients with improved, unchanged, or deteriorated performance differed significantly between the groups with and without acromegaly (Fisher exact test; $p=0.028$ ). Within groups, olfactory performance improved significantly in patients with acromegaly (paired Wilcoxon test; $p=0.009$ ), but not in patients without the disease. 


\section{Septal Perforation}

Among the 96 patients, we found 2 perforations at the time of surgery: one was in a patient with known cocaine abuse, and the other was in a patient who had undergone 3 prior TSSs at other centers. The other 94 patients did not develop any perforations within the follow-up time.

\section{Epistaxis Requiring Coagulation}

We encountered severe or persistent epistaxis in 3 (3\%) of 96 patients, who required transnasal surgical revision with coagulation of the sphenopalatine artery. All 3 had undergone TSS for the first time.

\section{Olfactory Performance}

A remarkable difference was found between patients with and without acromegaly in regard to olfactory performance. In patients with acromegaly, $65 \%$ improved in olfactory performance, versus $44 \%$ of those without acromegaly, and only $13 \%$ of patients with acromegaly deteriorated, versus $42 \%$ of those without the disease (Fig. 1). We propose 2 mechanisms by which this improvement may occur. First, an entrapment mechanism in the olfactory system, analogous to median nerve neuropathy, ${ }^{7}$ may be reduced after successful treatment of acromegaly, leading to improved nerve function. Second, excessive GH production in patients with acromegaly is associated with hypertrophy of the nasal mucosa,${ }^{11}$ which consecutively congests the region below the cribriform plate. Reduced GH production after surgery may reverse this effect, thus enabling an improved interaction of aromatic molecules with the primary sensory neurons. This hypothesis is supported by the fact that 18 patients with acromegaly were cured, 4 had normal GH serum levels, and only 1 presented with persistent elevation of GH levels.

There is a limitation of our study in that we used the Sniffin' Sticks olfactory test to assess the patients preoperatively and the Zürcher Geruchstest for postoperative follow-up. The change was motivated by the better robustness of the latter test in our follow-up setting. To compare between test results, we had to calculate percentages from the test results instead of using the scored number values. As a consequence, we confined the results to only 3 broad groups: normosmic, hyposmic, and anosmic, without trying to distinguish more subtle nuances in olfactory performance.

Comparison of our data on olfactory complications with existing literature is difficult, because olfactory performance has hardly ever been systematically observed in patients who have undergone TSS. Higgins et al. ${ }^{6}$ mention a 2\%-12\% incidence of anosmia and hyposmia in patients treated with the transseptal approach. Koren et al. ${ }^{8}$ found hyposmia and anosmia in $2(10 \%)$ of 20 patients who had undergone transnasal endoscopic surgery, and Tan and Jones ${ }^{12}$ report on 1 patient (4\%) of 25 with hyposmia. Our results, with a $35 \%$ prevalence of postoperative olfactory deterioration, are based on the largest series to date. It must be considered that this high prevalence also accounts for subtle deterioration within the normosmic range, and only 6 patients were actually anosmic.

\section{Nasal Airway Patency and Other Complications}

Fairley et al. ${ }^{4}$ have demonstrated a strong correlation between the subjective sensation of nasal patency and the objective nasal inspiratory peak flow rate. Considering this, we conducted a survey for postoperative reduced nasal airway patency, and found it in $8 \%$ of patients by questioning them. These results were similar to those of Tan and Jones,${ }^{12}$ who analyzed peak nasal inspiratory flow rates and found $3(12 \%)$ of 25 patients with reduced nasal air passage. Griffith and Veerapen, ${ }^{5}$ the pioneers of the direct transnasal approach, observed a 3\% diminishment of postoperative nasal breathing capability in their cohort of 100 patients. Again, in the analysis of this particular nasal complication, we registered a more than proportional percentage of patients with acromegaly (55\%) among those who improved in nasal respiration. This further fortifies our assumption of decreasing mucosal hypertrophy after normalization of $\mathrm{GH}$ production as one of the factors leading to improvement of nasal function in these patients. Furthermore, we postulate that the choice in the side of direct transnasal access may be beneficial for patients who present with septal deviation. If the speculum is placed on the side that has greater obstruction due to the septal deviation, the amount of deviation may in part be corrected as a side effect of the direct transnasal approach.

No new perforations of the nasal septum as a result of the direct transnasal approach were observed. This finding is comparable with prior studies in which the direct transnasal microsurgical resection of pituitary adenomas has been analyzed..$^{3,12}$ In contrast to this, several authors applying an endoscopic approach reported a 5\%-10\% septal perforation rate. ${ }^{6,8}$ As mentioned by others, we suspect that this may be due to the increased mechanical manipulation within the nasal cavities during endoscopic pituitary surgery.

With a rate of approximately $3 \%$ for epistaxis requiring revision surgery, this complication should not be underestimated. In all cases of epistaxis, hemorrhage occurred from branches of the sphenopalatine artery. Management consisted of monopolar cauterization of the bleeding artery in the sphenopalatine foramen, which in all cases was conducted successfully. Published rates of epistaxis range from $1 \%$ to $4 \%$., ${ }^{9}$

Prior TSS performed via varying approaches does not present a contraindication for repeat direct transnasal TSS in cases of recurrent disease. In all of our 16 cases with prior TSS, in some of which the patients have undergone multiple operations (Table 1), the procedure was always accomplished successfully without a higher occurrence of nasal complications.

\section{Conclusions}

Our data provide the first significant evidence for improvement of olfactory performance in patients with acromegaly after TSS of GH-producing adenomas. Furthermore, postoperative olfactory disturbance in transnasal TSS is more frequent than previously reported, when also accounting for subtle deterioration within the normosmic range. Nevertheless, recurrent transnasal TSS can be per- 


\section{Improved olfaction after transsphenoidal surgery in acromegaly}

formed successfully, even multiple times, and does not involve a higher risk of nasal complications.

\section{Disclosure}

The authors report no conflict of interest concerning the materials or methods used in this study or the findings specified in this paper.

Author contributions to the study and manuscript preparation include the following. Conception and design: Bernays, Holzmann. Acquisition of data: Bernays, Actor, Prömmel, Holzmann. Analysis and interpretation of data: Bernays, Actor, Sarnthein. Drafting the article: Bernays, Actor, Sarnthein. Critically revising the article: Bernays, Actor, Sarnthein, Holzmann. Reviewed final version of the manuscript and approved it for submission: Bernays, Actor, Sarnthein. Statistical analysis: Actor, Sarnthein. Study supervision: Bernays.

\section{Acknowledgment}

The authors thank Sarah R. Haile, Ph.D., for her expert assistance with the statistical analysis.

\section{References}

1. Bonadonna S, Doga M, Gola M, Mazziotti G, Giustina A: Diagnosis and treatment of acromegaly and its complications: consensus guidelines. J Endocrinol Invest 28 (11 Suppl International):43-47, 2005

2. Buck LB: The olfactory multigene family. Curr Opin Neurobiol 2:282-288, 1992

3. Cooke RS, Jones RA: Experience with the direct transnasal transsphenoidal approach to the pituitary fossa. Br J Neurosurg 8:193-196, 1994
4. Fairley JW, Durham LH, Ell SR: Correlation of subjective sensation of nasal patency with nasal inspiratory peak flow rate. Clin Otolaryngol Allied Sci 18:19-22, 1993

5. Griffith HB, Veerapen R: A direct transnasal approach to the sphenoid sinus. Technical note. J Neurosurg 66:140-142, 1987

6. Higgins TS, Courtemanche C, Karakla D, Strasnick B, Singh RV, Koen JL, et al: Analysis of transnasal endoscopic versus transseptal microscopic approach for excision of pituitary tumors. Am J Rhinol 22:649-652, 2008

7. Jenkins PJ, Sohaib SA, Akker S, Phillips RR, Spillane K, Wass JA, et al: The pathology of median neuropathy in acromegaly. Ann Intern Med 133:197-201, 2000

8. Koren I, Hadar T, Rappaport ZH, Yaniv E: Endoscopic transnasal transsphenoidal microsurgery versus the sublabial approach for the treatment of pituitary tumors: endonasal complications. Laryngoscope 109:1838-1840, 1999

9. Marquardt G, Yahya H, Hermann E, Seifert V: Direct transnasal approach for pituitary surgery. Neurosurg Rev 27:83-88, 2004

10. Melmed S, Casanueva FF, Cavagnini F, Chanson P, Frohman L, Grossman A, et al: Guidelines for acromegaly management. J Clin Endocrinol Metab 87:4054-4058, 2002

11. Skinner DW, Richards SH: Acromegaly-the mucosal changes within the nose and paranasal sinuses. J Laryngol Otol 102:1107-1110, 1988

12. Tan LK, Jones RA: Nasal complications of the direct transnasal approach to the pituitary fossa. Br J Neurosurg 9:739_ 742,1995

Manuscript submitted June 15, 2010.

Accepted July 16, 2010.

Address correspondence to: René L. Bernays, M.D., Department of Neurosurgery, University Hospital Zurich, Frauenklinikstrasse 10, 8091 Zurich, Switzerland. email: rene.bernays@usz.ch. 